\title{
Olfaction in Neurologic and Neurodegenerative Diseases: A Literature Review
}

\author{
Maria Dantas Costa Lima Godoy ${ }^{1}$ Richard Louis Voegels ${ }^{1}$ Fábio de Rezende Pinna ${ }^{1}$ Rui Imamura ${ }^{1}$ \\ José Marcelo Farfel² \\ 1 Department of Otorhinolaryngology, Faculdade de Medicina da \\ Universidade de São Paulo, São Paulo, São Paulo, Brazil \\ 2 Department of Geriatrics, Faculdade de Medicina da Universidade de \\ São Paulo, São Paulo, Brazil

\begin{abstract}
Address for correspondence Maria Dantas Costa Lima Godoy, MD, Department of Otorrinolaringologia, Faculdade de Medicina da Universidade de São Paulo, Rua Dr. Eneas de Carvalho Aguiar, 255, 6 andar, 6167 Cerqueira Cesar, São Paulo 05403000, Brazil
\end{abstract} \\ (e-mail: mariadcostalima@hotmail.com).
}

Int Arch Otorhinolaryngol 2015;19:176-179.

\begin{abstract}
Introduction Loss of smell is involved in various neurologic and neurodegenerative diseases, such as Parkinson disease and Alzheimer disease. However, the olfactory test is usually neglected by physicians at large.

Objective The aim of this study was to review the current literature about the relationship between olfactory dysfunction and neurologic and neurodegenerative diseases.

Keywords

- olfactory mucosa

- cerebrum

- dementia

- olfaction disorders

- aging

Data Synthesis Twenty-seven studies were selected for analysis, and the olfactory system, olfaction, and the association between the olfactory dysfunction and dementias were reviewed. Furthermore, is described an up to date in olfaction.

Conclusion Otolaryngologist should remember the importance of olfaction evaluation in daily practice. Furthermore, neurologists and physicians in general should include olfactory tests in the screening of those at higher risk of dementia.
\end{abstract}

\section{Introduction}

Olfaction is usually neglected by physicians at large, even though it monitors the intake of airborne agents into the human respiratory system, including dangerous substances, and warns of spoiled foods. Furthermore, this primary sensory system enhances quality of life by adding to flavor and palatability of foods and beverages. ${ }^{1}$ Most complaints of decreased "taste" function actually reflect decreased smell function. ${ }^{1,2}$ In addiction, loss of smell is involved in various neurologic and neurodegenerative diseases, such as Parkinson disease (PD), Alzheimer disease (AD), multiple sclerosis, and Huntington disease. ${ }^{3}$

The aim of this study was to review the current literature about the relationship between olfactory dysfunction and neurologic and neurodegenerative diseases.

received

October 16, 2013

accepted after revision

July 27, 2014

published online

November 14, 2014
DOI http://dx.doi.org/ 10.1055/s-0034-1390136. ISSN $1809-9777$.

\section{Review of the Olfactory System}

\section{Olfactory Mucosa Cellular Composition and Neurogenesis}

Human olfactory mucosa is a pseudostratified columnar epithelium resting on a highly cellular lamina propria. Four types of cells are present at the epithelium: ciliated bipolar olfactory receptors, sustentacular cells, microvillar cells, and basal cells, beyond the Bowman gland. ${ }^{1,4-6}$

The olfactory system is composed by peripheral structures of $\sim 6$ million bipolar receptor cells located within the olfactory neuroepithelium. ${ }^{4,7}$ This ciliated bipolar olfactory receptor cell is a true bipolar neuron, projecting a single dendrite to the surface of the olfactory neuroepithelium and a single axon to the olfactory bulb. The dendrite extends to the epithelial surface and has nonmotile cilia with membrane receptors,

Copyright $\odot 2015$ by Thieme Publicações License terms Ltda, Rio de Janeiro, Brazil

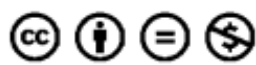


where odor molecules bind. ${ }^{4}$ The axons form into bundles, called the olfactory fila, and project through the cribriform plate of the ethmoid bone and synapse at the olfactory bulb. In humans, the surface area of the cilia is $\sim 25 \mathrm{~mm}$. ${ }^{1,8}$

Olfactory receptor neurons are surrounded by the sustentacular cells, which probably contribute to regulation and conservation of the appropriate ionic environment around the receptor neurons for adequate olfactory transduction. ${ }^{1,4}$

Microvillar cells presumably act as a second morphologically distinct class of chemoreceptor, first described in 1982. However, their precise role in olfaction has not yet been clearly demonstrated. ${ }^{4,9}$

The Bowman gland is a serous-producing tubuloalveolar gland, another component of the lamina propria, composed of secretory acini with a duct that passes through the olfactory epithelium. ${ }^{4,10}$ This secretion probably is essential for olfactory transduction. ${ }^{4}$

Recent studies have shown that the olfactory system displays robust and functional neurogenesis throughout life, ${ }^{6}$ by containing cells with very broad developmental potency. Human olfactory mucosa yield neurospheres that could be propagated as secondary and tertiary neurospheres. ${ }^{11}$ They are the basal cells, a well-recognized distinct population of stem cells of the olfactory epithelium, capable of continuously regenerating olfactory receptor neurons throughout the life span. ${ }^{4,11,12}$ The basal cells have multipotency and self-renewal characteristics and are able to give rise experimentally to other neural and non-neural cells. ${ }^{4,6,13}$

\section{Olfactory Bulb and Primary Olfactory Cortex}

As described previously, the single axons of the 10 to 20 million olfactory receptor neurons join together into fascicles and nerves, which pass through the 15 to 20 foramina of each cribriform plate to synapse within the olfactory bulb. ${ }^{4,14}$

The olfactory bulb continues to the olfactory tract and arrives at the primary olfactory cortex areas, such as anterior olfactory nucleus, entorhinal cortex, and amygdale. ${ }^{15,16}$

\section{Olfactory Mucosa Localization}

Theoretically, the human olfactory mucosa constitutes $1.25 \%$ of the nasal mucosa, corresponding to an area of $\sim 2 \mathrm{~cm}^{2}$. The number and density of bipolar olfactory neurons are probably $\sim 6 \times 10^{6}$ and $30,000 / \mathrm{mm}^{2}$, respectively. ${ }^{4,17}$

The olfactory epithelium in the human fetus is uniformly distributed without interruption by respiratory epithelium in a continuous pattern. ${ }^{18}$ Féron and other authors have observed that the distribution of the olfactory epithelium in adult humans is frequently disrupted with interspersed patches of respiratory epithelium. ${ }^{18-20}$ Comparison of fetal and adult samples suggests that invasion of respiratory tissue into olfactory epithelium increases with age, as suggested in a previous study of adult olfactory epithelium. ${ }^{18}$ This probably occurs because this mucosa is a dynamic structure with features reflecting innate and environmental as well as developmental influences. Thus, it can be assumed that the precise location and the overall dimension of the neuroepithelium may be different among individuals and may change over time. ${ }^{4}$
Recent anatomical studies modified the previous concept of distribution area of the olfactory mucosa. It was assumed that the neuroepithelium was restricted to the area of the cribriform plate, superior turbinate, and the opposite superior nasal septum. ${ }^{4}$ Leopold and other authors demonstrated that the olfactory mucosa extends within the medial and anterior surface of the middle turbinate, either in the lateral or in the medial wall of the nasal cavity. ${ }^{21,22}$ This would not be in conflict with the work of Biedlingmaier and Whelan ${ }^{23}$, since they looked at much more inferior middle turbinate tissue and did not find olfactory tissue. ${ }^{21}$

\section{Discussion}

\section{Olfactory Deficit in Elderly People}

A decay of the smell function occurs in old age. In fact, age is the strongest correlate of olfactory decline in healthy adult humans and has a much larger impact than even cigarette smoking. ${ }^{1}$ These data are confirmed by cross-sectional and longitudinal studies. ${ }^{24}$

Generally, olfactory impairment related to age is more severe for men than for women, although individual differences are present. This deficit often goes unnoticed and is rarely investigated by physicians, unlike alterations in hearing and vision. About $2 \%$ of the population under 65 years of age has olfactory impairment. This rises dramatically between 65 and 80 years, with about half of the population complaining of loss of smell. Over 80 years, smelling problems are noticed by $75 \%$ of elderly. ${ }^{1}$

Possible reasons for smell changes related to age include ossification and closure of the foramina of the cribriform plate, age-related degenerative processes occurring in the brain, ${ }^{1,24}$ and cumulative damage to the olfactory receptors from different types of insults throughout life. ${ }^{1}$

\section{Olfactory Deficit and Dementia}

Recently, the olfactory neuroepithelium has attracted the renewed interest of scientists, because the olfactory mucosa has the potential to be an early marker of neurodegenerative conditions, such as schizophrenia, $\mathrm{AD}$, multiple sclerosis, and PD. ${ }^{4}$

There is considerable variation in the prevalence and magnitude of olfactory dysfunction among neurodegenerative diseases. In AD, PD, and Parkinson-dementia complex of Guam, olfactory dysfunction is severe (University of Pennsylvania Smell Inventory Test [UPSIT] scores under 20), whereas that of Huntington disease, multi-infract dementia, amyotrophic lateral sclerosis, and schizophrenia is more moderate. Progressive supranuclear palsy is associated with minor changes in olfactory function, even though it shares major clinical features with PD. These data suggest that olfactory testing could help in differential diagnosis of several neurodegenerative diseases. ${ }^{1}$

Olfactory deficit can be noticed in the ability to detect, recognize, and remember odorants in the elderly, particularly in patients with $\mathrm{AD} .^{25}$ In the case of $\mathrm{AD}$, smell problems occur in the beginning of the disease, ${ }^{26}$ and this pattern can reflect an "preclinical" period of disease development by preceding 
the onset of classic disease symptoms. ${ }^{1}$ Moreover, olfactory impairment is mostly found in individuals at risk for $A D$, including subjects with mild cognitive impairment who eventually develop $A D$, those with another potential risk factor for AD (namely subjective memory complaints), ${ }^{25,27}$ and relatives of $\mathrm{AD}$ patients. ${ }^{7,27,28}$ Olfactory dysfunction in $\mathrm{AD}$ is associated with disease progression, can be helpful in the differential diagnosis of major depression and AD, and may have clinical value as an early diagnostic marker in predicting incident $\mathrm{AD}$ in high-risk individuals. ${ }^{25}$ The presence of apolipoprotein E-4 allele in an anosmic normal individual increases the risk of having cognitive decline in the future by 4.9 -fold. ${ }^{1}$

Surprisingly, most patients with AD and PD are unaware of their olfactory loss before taking the test; 85 to $90 \%$ of patients in the early stages present olfactory impairment, and this deficit is associated with decreased activation of central odor processing structures (as measured by functional imaging). ${ }^{1,15}$

In $A D$, neuropathologic changes within olfaction-related brain regions usually accompany these olfactory changes. One of the pathologic hallmarks of $\mathrm{AD}$, the neurofibrillary tangles (NFTs), have been identified within the olfactory bulb, olfactory tract, transentorhinal and entorhinal cortex, anterior olfactory nucleus, and amygdale. ${ }^{25}$ The number of NFTs within such regions has been correlated with the severity of dementia. ${ }^{15}$ Recent neuropathologic studies suggest that AD-related pathology may begin within olfactory central cortex and then spread to multiple areas of the brain. ${ }^{15}$ These neuropathologic changes have been associated with impaired olfaction around the time of death in those without dementia or with mild cognitive impairment. ${ }^{24,27}$

As seen, olfactory impairment has been significantly associated with the AD neuropathology burden in the brain and the risk of future AD. Some animal models of AD-related neuropathologic changes have indicated a strong association between NFT in the olfactory system and cognitive decline and a negative association between amyloid- $\beta$ burden in the brain, another hallmark of AD disease, and olfaction. Moreover, oxidative damage in the olfactory epithelium is present in the early stages of $\mathrm{AD} .^{27}$

\section{Functional Magnetic Resonance Imaging in Patients with AD}

Wang demonstrated that functional magnetic resonance imaging (fMRI) is sensitive to changes in olfactory function due to AD. Patients with AD have reduced BLOD (bloodoxygen-level dependent) signals in the hippocampus and insula regions when compared with healthy control subjects of similar age. Such alterations are significantly correlated with UPSIT, Mini-Mental State Examination, and CDR (clinical dementia rating) scores, proposing the significance of olfactory fMRI in patients with AD. ${ }^{15}$

Furthermore, when the odorant concentration is increased 10 -fold, only slightly more activation is induced in some brain regions. These data about additional recruitment of activity suggest that total anosmia in $\mathrm{AD}$ is not the rule but rather the exception, and they indicate that at least some residual capacity is available. ${ }^{15,26}$ Experimental results indicate the feasibility for using olfactory fMRI as a marker for diagnosis and evaluation of AD. ${ }^{15}$

\section{Olfactory Epithelium Biopsy}

Because the olfactory mucosa contains the only surface neural cells of the body, the olfactory receptor neurons, some authors call the neuroepithelium the "window to the brain." Therefore, an olfactory biopsy could potentially help to understand what occurs in the brain with neurologic and neurodegenerative disease. ${ }^{4}$ Olfactory epithelium is accessible for low-risk biopsy, allowing examination of the neurogenesis process, ${ }^{6,18}$ as shown by neuropathologic studies on neurodegenerative diseases like $\mathrm{AD}$ and $\mathrm{PD} .^{18}$

\section{Gender Differences in Human Olfactory Bulb}

Recent studies indicate the existence of differences in human olfactory bulb related to gender. Oliveira-Pinto et al demonstrate a sex-related difference in the absolute number of total, neuronal and non-neuronal cells, favoring women by $40-50 \%$, which may have olfactory functional impact. ${ }^{29}$

\section{Conclusions}

Olfactory dysfunction can manifest in various degrees and difficulties in discrimination, odor identification, and olfactory memory. Neurologic and neurodegenerative diseases, particularly AD and PD, are major causes of dysosmia. There is much evidence that olfactory tests can be used to differentiate diagnosis between PD and other types of parkinsonism.

This review shows that olfactory tests can be a useful tool in differential diagnosis of dementia and other diseases, as well as among the various types of dementia, and indicates that impairment in olfactory discrimination can predict future cognitive decline.

However, some questions remain unanswered: 'What is the boundary between the olfactory changes related to aging and those caused by disease?'; 'Is it possible to differentiate them through the olfactory tests?'; 'In which time the pathological changes of $\mathrm{AD}$ are installed in the olfactory structures?'. More studies are necessary to clarify these issues..

Based on current knowledge of the association between smell and dementias, the authors suggest that the otolaryngologist should remember the importance of olfaction evaluation in daily practice. Furthermore, neurologists and physicians in general should all include olfactory tests in the screening those at higher risk of dementia.

\section{References}

1 Doty RL. The olfactory system and its disorders. Semin Neurol 2009;29(1):74-81

2 Deems DA, Doty RL, Settle RG, et al. Smell and taste disorders, a study of 750 patients from the University of Pennsylvania Smell and Taste Center. Arch Otolaryngol Head Neck Surg 1991;117(5): 519-528

3 Doty RL. Clinical studies of olfaction. Chem Senses 2005;30 (Suppl 1):i207-i209 
4 Escada PA, Lima C, da Silva JM. The human olfactory mucosa. Eur Arch Otorhinolaryngol 2009;266(11):1675-1680

5 Hahn CG, Han LY, Rawson NE, et al. In vivo and in vitro neurogenesis in human olfactory epithelium. J Comp Neurol 2005; 483(2):154-163

6 Ronnett GV, Leopold D, Cai X, et al. Olfactory biopsies demonstrate a defect in neuronal development in Rett's syndrome. Ann Neurol 2003;54(2):206-218

7 Doty RL, Perl DP, Steele JC, et al. Olfactory dysfunction in three neurodegenerative diseases. Geriatrics 1991;46(Suppl 1):47-51

8 Graves AB, Bowen JD, Rajaram L, et al. Impaired olfaction as a marker for cognitive decline: interaction with apolipoprotein $\mathrm{E}$ epsilon4 status. Neurology 1999;53(7):1480-1487

9 Montani G, Tonelli S, Elsaesser R, Paysan J, Tirindelli R. Neuropeptide $\mathrm{Y}$ in the olfactory microvillar cells. Eur J Neurosci 2006;24(1): 20-24

10 Moulton DG, Beidler LM. Structure and function in the peripheral olfactory system. Physiol Rev 1967;47(1):1-52

11 Murrell W, Féron F, Wetzig A, et al. Multipotent stem cells from adult olfactory mucosa. Dev Dyn 2005;233(2):496-515

12 Bartolomei JC, Greer CA. Olfactory ensheathing cells: bridging the gap in spinal cord injury. Neurosurgery 2000;47(5):1057-1069

13 Roisen FJ, Klueber KM, Lu CL, et al. Adult human olfactory stem cells. Brain Res 2001;890(1):11-22

14 Hadley K, Orlandi RR, Fong KJ. Basic anatomy and physiology of olfaction and taste. Otolaryngol Clin North Am 2004;37(6): 1115-1126

15 Wang J, Eslinger PJ, Doty RL, et al. Olfactory deficit detected by fMRI in early Alzheimer's disease. Brain Res 2010;1357:184-194

16 Attems J, Jellinger KA. Olfactory tau pathology in Alzheimer disease and mild cognitive impairment. Clin Neuropathol 2006; 25(6):265-271

17 Moran DT, Rowley JC III, Jafek BW, Lovell MA. The fine structure of the olfactory mucosa in man. J Neurocytol 1982;11(5):721-746
18 Féron F, Perry C, McGrath JJ, Mackay-Sim A. New techniques for biopsy and culture of human olfactory epithelial neurons. Arch Otolaryngol Head Neck Surg 1998;124(8):861-866

19 Morrison EE, Costanzo RM. Morphology of the human olfactory epithelium. J Comp Neurol 1990;297(1):1-13

20 Kobal G, Van Toller S, Hummel T. Is there directional smelling? Experientia 1989;45(2):130-132

21 Leopold DA, Hummel T, Schwob JE, Hong SC, Knecht M, Kobal G. Anterior distribution of human olfactory epithelium. Laryngoscope 2000;110(3 Pt 1):417-421

22 Nibu K, Li G, Zhang X, et al. Olfactory neuron-specific expression of NeuroD in mouse and human nasal mucosa. Cell Tissue Res 1999; 298(3):405-414

23 Biedlingmaier JF, Whelan P, Zoarski G, Rothman M. Histopathology and CT analysis of partially resected middle turbinates. Laryngoscope 1996;106(1 Pt 1):102-4

24 Wilson RS, Yu L, Schneider JA, Arnold SE, Buchman AS, Bennett DA. Lewy bodies and olfactory dysfunction in old age. Chem Senses 2011;36(4):367-373

25 Peters JM, Hummel T, Kratzsch T, Lötsch J, Skarke C, Frölich L. Olfactory function in mild cognitive impairment and Alzheimer's disease: an investigation using psychophysical and electrophysiological techniques. Am J Psychiatry 2003;160(11):1995-2002

26 Doty RL. Studies of human olfaction from the University of Pennsylvania Smell and Taste Center. Chem Senses 1997;22(5): 565-586

27 Sohrabi HR, Bates KA, Weinborn MG, et al. Olfactory discrimination predicts cognitive decline among community-dwelling older adults. Transl Psychiatr 2012;2:e118

28 Doty RL. The olfactory vector hypothesis of neurodegenerative disease: is it viable? Ann Neurol 2008;63(1):7-15

29 Oliveira-Pinto AV, Santos RM, Coutinho RA, et al. Sexual dimorphism in the human olfactory bulb: females have more neurons and glial cells than male. Plos One (In Press) 